\title{
Um estudo dos modelos de geração interna do campo geomagnético
}

\author{
A STUDY OF THE MODELS OF INTERNAL GENERATION OF THE GEOMAGNETIC FIELD
}

\author{
Daniel Seabra Nogueira Alves Albarelul1, Emilson Pereira Leite².
}

1 - Instituto de Pesquisas Tecnológicas do Estado de São Paulo, São Paulo SP. dseabra@ipt.br

2 - Depto. Geologia e Recursos Naturais, Instituto de Geociências, Universidade Estadual de Campinas, Campinas SP. emilson@ige.unicamp.br

ABSTRACT: One of the main challenges of global geophysics is to understand how the dynamics of the external liquid nucleus of the earth maintains the geomagnetic field, using geodynamo theories. The geomagnetic field is of extreme importance for sustaining life on Earth, since it protects us from high energy particles emanating from the sun, besides being useful for navigation. Because it is inaccessible to man, studies of the nucleus are based on results obtained from models in laboratories and computers, that simulate conditions and parameters of the interior of the planet. Each model has its singularities, similarities and restrictions. Thus, this article gathers basic information about how the published geodynamo models work. A review of international articles and books on the subject was conducted, in order to compile, organize and summarize that information. Concepts, theories and the main equations that describe those models are explained, and statistical analyses are presented for the main parameters utilized by the models.

\section{Manuscrito:}

Recebido: 05/11/2015

Corrigido: $30 / 09 / 2016$

Aceito: 04/10/2016

Citation: Albarelli D.S.N.A., Leite E.P.. 2016. Um estudo dos modelos de geração interna do campo geomagnético. Terræ Didatica, 12(3):172-183. <http://www. ige.unicamp.br/terraedidatica/ $>$.

Keywords: geomagnetic field, geodynamo models, magnetohydrodynamic (MHD)

\section{Introdução}

A observação de que a Terra possui um campo magnético é muito antiga, remontando à antiguidade clássica. Um dos primeiros trabalhos publicados sobre o assunto, De Magnete, foi feito por William Gilbert no inicio do século XVII, reunindo aspectos qualitativos sobre o magnetismo terrestre (Lowrie 2007). Em 1838, a partir das medidas das componentes do campo magnético coletadas até o momento na superfície da Terra e da análise do potencial magnético, Gauss forneceu suporte quantitativo para os trabalhos de Gilbert, provando que a origem para o campo provinha em sua grande maioria do interior do planeta (Glatzmaier \& Roberts 1997). Anos mais tarde Joseph Lamor em 1919, baseado em seus estudos com o Sol, propôs que um dínamo fluido autossustentável seria responsável pela geração do campo magnético do Sol e possivelmente de outros planetas como a Terra. Sua teoria ganhou mais força com a descoberta, em 1926, de Harold Jeffreys de que o núcleo externo da Terra era composto de ferro liquido (Davidson 2001). A Figura 1 ilustra distintas camadas do interior da Terra, incluindo o núcleo externo, onde padrões complexos de circulação de fluidos geram o campo geomagnético.
Um dínamo fluido autossustentável, no caso da Terra um geodínamo, é um mecanismo onde um fluido condutor em movimento (ferro liquido no núcleo externo) produz correntes elétricas capazes de gerar linhas de campo magnético. Estas, por sua vez, são esticadas e torcidas pela movimentação do fluido e pela rotação da Terra, reforçando o campo magnético original por um processo de indução eletromagnética (Davidson 2001). A energia responsável pela movimentação do fluido é a convecção termal e composicional sugeridas respectivamente por Verhoogen em 1961 e Braginsky em 1963 (apud Glatzmaier \& Roberts 1998).

Em 1934, Thomas Cowling propôs um teorema em que um dínamo não pode manter um campo magnético com simetria axial, semelhante ao campo poloidal observado na superfície da Terra. Por outro lado, anos mais tarde em 1958, George Backus e Arvid Hezenberg provaram independentemente que um dínamo pode manter um campo magnético simétrico autossustentável, embora tenham utilizado alguns parâmetros geofísicos posteriormente considerados pouco realísticos (Glatzmaier \& Roberts 1997). Os próximos trabalhos utilizaram parâmetros geofísicos mais realísticos, tentando solucionar as equações para dínamos cinemáticos. 


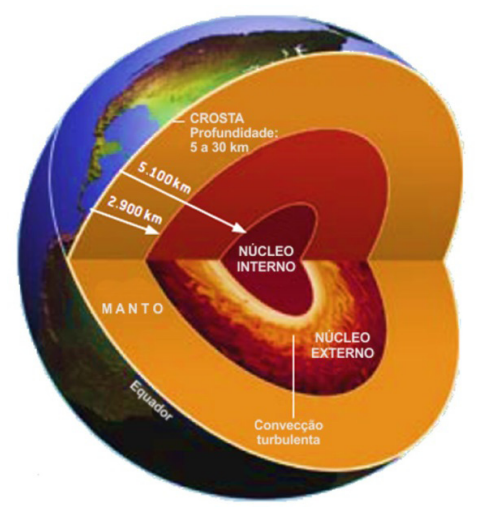

Figura 1. O interior da Terra dividido em quatro camadas: crosta, manto, núcleo externo e núcleo interno. 0 movimento turbulento de um grande volume de líquido condutor de eletricidade no núcleo externo gera o campo geomagnético. Fonte: Glatzmaier \& Olson (2005)

A próxima etapa foi entender não só quais fluidos geravam campos magnéticos, mas também como era a dinâmica desses fluidos através de modelos em magnetohidrodinâmica.

É importante ressaltar que as equações envolvidas nos modelos de geodínamo, as quais são apresentadas e discutidas neste artigo, são extremamente difíceis de serem resolvidas. Na prática, a capacidade computacional é um fator preponderante no realismo dessas soluções. Atualmente, o modelo considerado mais realístico e que consegue reproduzir inclusive as reversões do campo geomagnético é aquele originalmente apresentado em Glatzmaier \& Roberts (1995a,b) e posteriormente discutido em Glatzmaier \& Roberts (1998) dentro de um contexto envolvendo outros quatro modelos.

\section{Modelos Cinemáticos}

\subsection{Equações e Teoria}

A questão central nestes modelos é entender em que situações uma dada corrente elétrica pode gerar um campo magnético (Sreenivasan 2010) e mantê-lo autossustentável, ou seja, sem que ele desapareça com o tempo (Glatzmaier \& Roberts 1998). Para isso, são estipulados valores para a velocidade do fluido e é verificado se existem soluções que satisfazem as equações eletrodinâmicas descritas abaixo (Equações 3 e 6). Neste modelo não se utiliza a dinâmica de fluidos e dessa forma suas características, tais como temperatura e flutuabilidade, são ignoradas. As equações eletrodinâmicas utilizadas são as equações pré-Maxwell (Equações 1, 2 e 3), expressas em suas formas diferenciais, e a lei de $\mathrm{Ohm}$ (4) para um condutor em movimento em um campo magnético, representando o ferro fundido no núcleo externo da Terra (Jacobs 1994):

$$
\begin{aligned}
& \nabla x \boldsymbol{E}=-\frac{\partial \boldsymbol{B}}{\partial t} \\
& \nabla x \boldsymbol{B}=\mu_{0} \boldsymbol{J} \\
& \nabla . \boldsymbol{B}=0 \\
& \boldsymbol{I}=\sigma(\boldsymbol{E}+\boldsymbol{V} x \boldsymbol{B})
\end{aligned}
$$

E e $\mathbf{B}$ representam respectivamente o campo elétrico e magnético, to tempo, $\mathbf{J}$ a densidade de corrente , $\mathbf{V}$ o campo de velocidade, $\mu_{0}$ a permeabilidade magnética ${ }^{1}$ e $\sigma$ a condutividade elétrica ${ }^{2}$. A Equação (1) é a Lei de Faraday da indução, a (2) é a Lei de Ampére e a (3) é a Lei de Gauss para campos magnéticos afirmando que monopólos magnéticos não existem (ver por ex. Halliday et al. 2007). Essas equações são ditas pré-Maxwell porque a corrente de deslocamento na Equação (2) é desprezada já que é muito pequena e pode ser negligenciada (Davidson 2001).

Ao substituir (4) em (2) e tomar o rotacional em ambos os lados, temos:

$$
\nabla x(\nabla x \boldsymbol{B})=\mu_{0} \sigma[\nabla x \boldsymbol{E}+\nabla x(\boldsymbol{V} x \boldsymbol{B})]
$$

$$
\begin{aligned}
& \text { Considerando que } \\
& \nabla x(\nabla x \boldsymbol{B})=\nabla(\nabla \cdot \boldsymbol{B})-\nabla^{2} \boldsymbol{B}=-\nabla^{2} \boldsymbol{B}
\end{aligned}
$$

e substituindo (1) em (5), obtemos a equação de indução magnética:

$$
\frac{\partial \boldsymbol{B}}{\partial t}=\nabla x(\boldsymbol{V} x \boldsymbol{B})+\eta \nabla^{2} \boldsymbol{B}
$$

Onde $\eta=\frac{1}{\mu_{0} \sigma}$ representa a difusividade magnética ${ }^{3}$. Esta é a principal equação utilizada nos modelos cinemáticos do geodínamo. Ela mostra que a evolução temporal e espacial do campo magnético no núcleo terrestre é dependente do termo de advecção, representado pelo primeiro termo à direita e pelo termo de difusão, o segundo à direita (Silva 2009). Se subtrairmos o termo de advecção, a equação (6) fica semelhante a uma equação de difusão, indicando que o campo magnético tende a decair por dissipa-

\footnotetext{
1 As altas temperaturas do núcleo terrestre sugerem uma permeabilidade magnética igual a do vácuo, ou seja, $\mu_{0}=4 \pi \times 10^{-7} \mathrm{H} / \mathrm{m}$ (Glatzmaier \& Roberts 1997)

$2 \sigma=(3-5) \times 10^{5} \Omega^{-1} \mathrm{~m}^{-1}$ no núcleo externo (Lowrie 2007).

$3 \eta \approx 2 \mathrm{~m}^{2} / \mathrm{s}$ no núcleo externo (Buffet 2002).
} 
ção ôhmica em aproximadamente $10^{4}$ anos (Buffet 2000). Se subtrairmos o termo de difusão, a equação

(6) representa a conversão de energia mecânica em energia magnética por uma força eletromotriz de módulo $|V \times B|$, criada quando um condutor com velocidade $\boldsymbol{V}$ se move em um campo magnético $\boldsymbol{B}$ (Glatzmaier \& Roberts 1997).

O que determina qual dos dois termos prevalecerá é o número magnético de Reynolds $\left(R_{m}\right)$, o qual representa a razão entre a advecção e a difusão (Davidson 2001):

$$
R_{m}=l \mathrm{v} / \eta=l \mathrm{v} \sigma \mu_{0}
$$

Onde $l$ tem dimensão de comprimento e $\mathrm{V}$ tem dimensão de velocidade. No caso do planeta Terra, $l$ é a diferença entre o raio do núcleo externo $\left(\mathrm{r}_{\mathrm{O}}\right)$ e o raio do núcleo interno $\left(\mathrm{r}_{\mathrm{i}}\right)$, os quais são respectivamente $3480 \mathrm{~km}$ e $1220 \mathrm{~km}$ (Lowrie 2007). Este elevado valor de $l$ garante $R_{m} \gtrsim 10$ (Jacobs 1994), e dessa forma a criação de linhas de campo magnéticas é muito superior a suas perdas por dissipação. Esse elevado valor permite usar o teorema do fluxo congelado de Alfvén para prever que as linhas de campo magnéticas são carregadas pelo ferro fundido como se elas estivessem congeladas nele, embora o núcleo externo não seja um condutor perfeito tal como predito por este teorema (Glatzmaier \& Roberts 1997).

\subsection{Eletrodinâmicas do campo médio}

Neste modelo de geodínamo, duas abordagens são utilizadas para explicar a cinemática da geração de campos magnéticos no núcleo terrestre, cada uma sob a perspectiva de uma escala particular. Em uma escala maior, o campo magnético poloidal é estirado pela rotação diferencial entre o núcleo interno sólido e o manto, originando um campo magnético toroidal, um mecanismo denominado de efeito $\omega$. As linhas do campo toroidal são paralelas ao fluido e à superfície do núcleo, por isso são confinadas a este e não podem ser medidas na superfície da Terra (Lowrie 2007). Já em uma escala menor, turbulências pequenas, helicoidais e assimétricas em relação ao eixo de rotação da Terra podem, aos poucos, esticar e encurvar as linhas de campo magnéticas toroidais até elas se tornarem poloidais de grande escala, em um mecanismo denominado de efeito $\alpha$. Esse campo poloidal é aquele que extravasa do núcleo, podendo ser observado e medido na superfície. Dessa forma, fecha-se o ciclo de regeneração de campos magnéti- cos autossustentáveis com a obtenção de um dínamo do tipo $\alpha \omega$ (Davidson 2001). O Teorema de Cowling é então refutado já que o efeito $\alpha$ permite a criação de um campo magnético simétrico (poloidal) em relação ao eixo de rotação da Terra a partir de dínamo com fluido em movimento assimétrico (turbulento).

Sob o ponto de vista dinâmico, a energia que abastece a rotação diferencial pode vir da convecção termal e composicional à medida que o núcleo interno cresce e libera calor latente e fluido rico em elementos leves (Davidson 2001). Embora haja evidencia sismológica de que a diferença de rotação entre o núcleo interno e o manto seja entre 0,3 e 3 graus por ano, ela não é conclusiva (Davidson 2001). Quanto às turbulências em pequena escala, não há evidências de quais seriam suas fontes de energia. Por outro lado, há outras teorias que desprezam essa separação entre pequena e grande escala.

\section{Modelos Turbulentos}

\subsection{Equações e Teoria}

Os modelos cinemáticos nos permitem entender quais correntes poderiam gerar um campo magnético. No entanto, ignoram o efeito que o campo magnético provoca no fluido condutor (Sreenivasan 2010). Para entender a evolução simultânea da velocidade e do campo magnético, se faz necessário incluir nos modelos a magnetohidrodinâmica, também conhecida como MHD: a interação entre correntes de fluidos eletricamente condutores (e não magnéticos) e campos magnéticos (Davidson 2001). Para isso é acrescentado aos modelos a equação de Navier-Stokes, obtida a partir da $2^{\text {a }}$ Lei de Newton para a conservação do momento (Kono \& Roberts 2002):

$\rho_{0}\left[\frac{\partial \boldsymbol{V}}{\partial t}+\boldsymbol{V} \cdot \nabla \boldsymbol{V}\right]=-\nabla P+\rho_{0} v \nabla^{2} \boldsymbol{V}+\rho \overline{\boldsymbol{g}}-2 \rho_{0} \boldsymbol{\Omega} \times \boldsymbol{V}+\boldsymbol{J} \times \boldsymbol{B}(8)$

Onde $\rho$ é a dersidade local, $\rho_{0}$ é a "densidade de referência”, $v$ é a viscosidade cinemática, $P=p-\frac{1}{2} \rho_{0}|\boldsymbol{\Omega} \times \boldsymbol{r}|^{2}$ é a pressão do fluido alterada pela aceleração centrifuga, $\boldsymbol{r}=|\boldsymbol{x}|$ onde $\boldsymbol{x}$ é um vetor que representa a distância até o geocentro, $\boldsymbol{\Omega}$ é a velocidade angular e $\overline{\boldsymbol{g}}$ a aceleração da gravidade. O lado esquerdo da equação (8) contém os termos da inercia linear e não linear, respectivamente, e ambas juntas fornecem a derivada convectiva ${ }^{4}$. Do lado

4 Derivada convectiva é a taxa de mudança da velocidade associada a um dado elemento do fluido. (Davidson 2001). 
direito estão todas as forças e parâmetros responsáveis pela mudança desse momento: o gradiente de pressão, a viscosidade, a flutuabilidade, a força de Coriolis devido a rotação e a força de Lorentz devido ao campo magnético. A maioria dos modelos de MHD (Kono \& Roberts 2002) usa nesta equação a aproximação de Boussinesq como premissa, ou seja, o fluido é tratado como incompressível com densidade dependendo linearmente apenas da temperatura e não da pressão. Dessa forma, $\rho=\rho_{0}\left[1-\beta\left(T-T_{0}\right)\right]$, onde $\beta$ é a expansividade termal (Kono $\&$ Roberts 2002 ) e a equação de conservação de massa, $\frac{\partial \rho}{\partial t}+\boldsymbol{\nabla}(\rho \boldsymbol{V})=0$, se reduz para a equação de continuidade (Davidson 2001) :

$$
\nabla \cdot \boldsymbol{V}=0(9)
$$

A força de Lorentz, $\mathbf{J} \times \mathbf{B}$, na equação de Navier-Stokes auxilia, sob um ponto de vista dinâmico, na compreensão de como o fluido amplifica as linhas de campo magnéticas, reforçando o campo magnético no núcleo da Terra. Esta força pode ser decomposta em uma pressão e tensão magnética. A pressão magnética não afeta o fluido, pois este é tomado como incompressível, no entanto a tensão magnética resiste ao estiramento provocado pelo fluido em movimento. Ao fazer esforço contra essa tensão, o fluido transforma parte de sua energia cinética em energia magnética para as linhas de campo (Hollerbach 1996). Como o geodínamo é movido pela convecção termal e composicional, também é necessário acrescentar a equ'çãa de flutuabilidade (Hollerbach 1996) onde $q$ é a difusividade termal ou molecular $\mathrm{e}^{\Theta}$ é a flutuabilidade:

$$
\left(\frac{\partial}{\partial t}+\boldsymbol{V} \cdot \nabla\right) \Theta=q \nabla^{2} \Theta
$$

A origem da flutuabilidade acontece pelo resfriamento do núcleo interno sólido ao longo da história geológica do planeta. A solidificação de ferro puro neste, libera simultaneamente calor latente e elementos leves para o núcleo externo, gerando convecção termal e composicional respectivamente.

Nos modelos de MHD de geodínamo, as Equações (3), (6), (8), (9) e (10) formam um conjunto de equações diferenciais não lineares, que são transformadas em adimensionais e posteriormente integradas no tempo, sendo os termos lineares tratados de forma implícita e os não lineares de forma explicita (Glatzmaier 2007). Dessa forma, é possível acompanhar a evolução em 3D do fluido

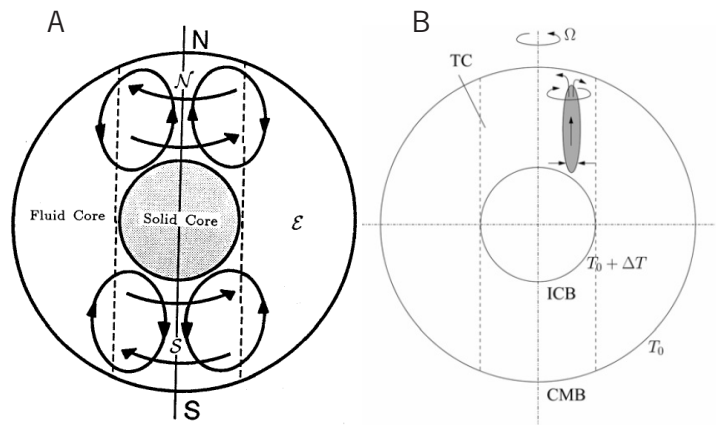

Figura 2. (A) Casca esférica entre o ICB e o CMB simulando a ascensão de uma pluma no núcleo externo da Terra nos modelos computacionais. Fonte: Sreenivasan (2010). (B) Esquema representativo das correntes meridionais e das correntes zonais que provavelmente existem no núcleo externo da Terra. A linha pontilhada representa o TC, a letra $\boldsymbol{\varepsilon}$ a parte externa ao TC. Solid Core é o núcleo sólido e Fluid Core é o núcleo líquido. Fonte: Glatzmaier \& Roberts (1997)

(núcleo externo liquido), do campo magnético e das transformações termodinâmicas relacionadas à convecção termal e composicional. De forma prática, isto é feito entre duas superfícies esféricas concêntricas, simulando a camada de transição entre o núcleo interno e o externo (do inglês $I C B^{5}$ ) ou $r_{i}$ e a camada limite entre o núcleo externo e o manto (do inglês $C M B^{6}$ ) ou $\mathrm{r}_{0}$ (Figura 2A). A proporção comumente usada nos modelos para $\mathrm{r} / \mathrm{r}_{\mathrm{o}}$ é 0,35 , semelhante àquela obtida a partir de dados sismológicos $\left(r_{i}=1220 \mathrm{~km} \mathrm{e} r_{o}=3480 \mathrm{~km}\right)$. A convecção termal se inicia aplicando-se uma temperatura maior, $\mathrm{T}_{0}+\Delta \mathrm{T}$, para a camada interna em relação à temperatura inicial, $\mathrm{T}_{0}$, da camada externa.

Uma vez iniciada a convecção, ela acontece de forma diferenciada dentro e fora do cilindro tangente (do inglês $T C^{7}$ ), um cilindro imaginário que toca o ICB no equador e é paralelo ao eixo de rotação da terra. Dentro do TC, o fluido sai do ICB e vai em direção ao CMB simetricamente em relação ao eixo de rotação da Terra e também paralelo a este na direção NS (Figura 2A). Ao chegar ao $\mathrm{CMB}$, o fluido é deslocado para longe do eixo de rotação e em seguida desce em direção ao ICB, também na direção NS. Ao chegar ao ICB, o fluido é reaproximado do eixo de rotação e fecha-se o ciclo de convecção dentro do TC. Tem-se então uma corrente meridional simétrica em relação ao eixo de rotação. No entanto, essa simetria é quebrada

\footnotetext{
$\overline{5 I C B}=$ Inner Core Boundary. É a fronteira entre o núcleo externo e o núcleo interno.

$6 C M B=$ Core Mantle Boundary. É a fronteira entre o núcleo externo e o manto. $7 \mathrm{TC}=$ Tangent Cylinder.
} 
pela ação da força de Coriolis, a qual torna o fluido helicoidal, deslocando a corrente meridional para oeste próximo ao $\mathrm{CMB}$ e para leste próximo ao ICB, tanto no hemisfério Norte (letra N) quanto no hemisfério Sul (letra S) (Figura 2B). Criam-se então correntes zonais não simétricas em relação ao eixo de rotação, mas que o circundam no ICB e CMB, também conhecidas como ventos termais (Glatzmaier \& Roberts 1997). Fora do cilindro tangente, a convecção acontece na forma de altas colunas e ocorre mais facilmente que dentro dele, pois a velocidade do fluido não varia na coordenada axial z devido ao estado geostrófico. Essa condição é atingida quando a força de Coriolis é equivalente ao gradiente de pressão na equação (8), desconsiderando todas as outras forças, admitindo viscosidade nula e um estado estacionário para o fluido (Sreenivasan 2010).

\subsection{Critérios utilizados nos modelos}

Os modelos de geodínamo em MHD utilizam alguns critérios semelhantes como as Equações (3) e (6), que descrevem o comportamento do campo magnético, e outros diferentes tais como variações de densidade, fontes de flutuabilidade e condições de contorno. Como citado no item 3.1, a maioria dos modelos utiliza a aproximação de Boussinesq, mas outros utilizam a aproximação inelástica (Kono \& Roberts 2002), considerando que a densidade na base do núcleo externo fluido é $20 \%$ maior que no topo (Glatzmaier 2007). Como fonte de flutuabilidade, o modelo pode utilizar a conveccão termal representada pela equação $\frac{\partial T}{\partial t}+\boldsymbol{V} \cdot \nabla \mathrm{T}=\kappa \nabla^{2} \mathrm{~T}+\epsilon$, ou a convecção composicional representada pela equação $\frac{\partial \xi}{\partial t}+\boldsymbol{V} . \nabla \xi=\kappa^{\xi} \nabla^{2} \xi+\epsilon^{\xi}$, onde $T$ é a tempe-

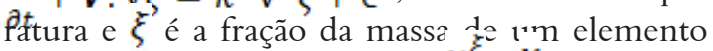
leve presente no ferro liquido, ${ }^{\xi} \mathrm{e}^{\kappa}$ são respectivamente a difusividade molecular e termal onde $c_{p}=\frac{k}{\rho c}$ sendo $k$ é a condutibilidade termal e $c_{p}=\frac{k}{\rho c_{x}}$ pacidade calorifica específica, $\epsilon$ é a uma fonte volumétrica de calor uniforme presente no núcleo ex+^ ${ }^{+}$no como por exemplo a radioatividade enquanto $\epsilon^{\xi}$ é uma fonte que representa o aumento na proporção do elemento leve no ferro liquido (Kono \& Roberts 2002).

As condições de contorno se referem às características do campo magnético e da velocidade do fluido nas camadas inferior e superior do núcleo externo, nas fronteiras com o núcleo interno e manto respectivamente. No caso da velocidade, a maioria dos modelos considera que as forças de viscosidade nas fronteiras são relevantes, de forma que a velocidade do fluido nelas seja zero ${ }^{8}$. Neste caso, o núcleo interno sólido pode ser escolhido de forma a rotacionar com a mesma velocidade angular que o manto ou não (Kono \& Roberts 2002). Já em outros modelos (Kono \& Roberts 2002) a influência dessa força sobre o fluido é reduzida, de modo que apenas a componente radial da velocidade seja zero9. Quanto às características do campo magnético, o manto pode ser considerado um isolante elétrico ou pode ser modelado como tendo uma fina camada na fronteira com o núcleo externo com as mesmas propriedades elétricas deste. De forma semelhante, o núcleo interno pode ser considerado como um isolante elétrico ou condutor parecido com o núcleo externo (Kono \& Roberts 2002). Mesmo com esses diferentes critérios, todos os modelos tem como resultado final duas características em comum que são o padrão dipolar do campo magnético fora do núcleo e o movimento para oeste das correntes zonais no CMB (Glatzmaier 2007) (Figura 2B).

\section{Dificuldades práticas, vantagens $e$ desvantagens}

Para simular a turbulência em modelos de geodínamo em três dimensões (3D) considerando valores reais para o raio e a taxa de rotação do núcleo, os pesquisadores são obrigados a usar valores muito mais altos para a viscosidade do fluido (difusividade viscosa) do que os valores possivelmente reais devido a limitações computacionais (Glatzmaier 2007). Isso resulta em uma grande desvantagem: o padrão de convecção do fluido se torna laminar de grande escala, como uma pluma se estendendo desde a base do núcleo externo até o seu topo. É uma desvantagem porque é mais provável que o núcleo externo contenha pequenos vórtices na escala de até algumas dezenas de metros e não plumas quilométricas (Glatzmaier \& Olson 2005) (Figura 3). A grande vantagem é que, neste tipo de modelo, o dínamo consegue ficar autossustentável.

Ao usar valores mais próximos da realidade para a viscosidade e as outras difusividades (térmica, composicional e magnética), são obtidos os vórtices e plumas de pequena escala nas fronteiras do núcleo. A desvantagem é que isso

80 termo usado em inglês para expressar essa condição é no-slip condition.

90 termo usado em inglês para expressar essa condição é stress-free condition. 

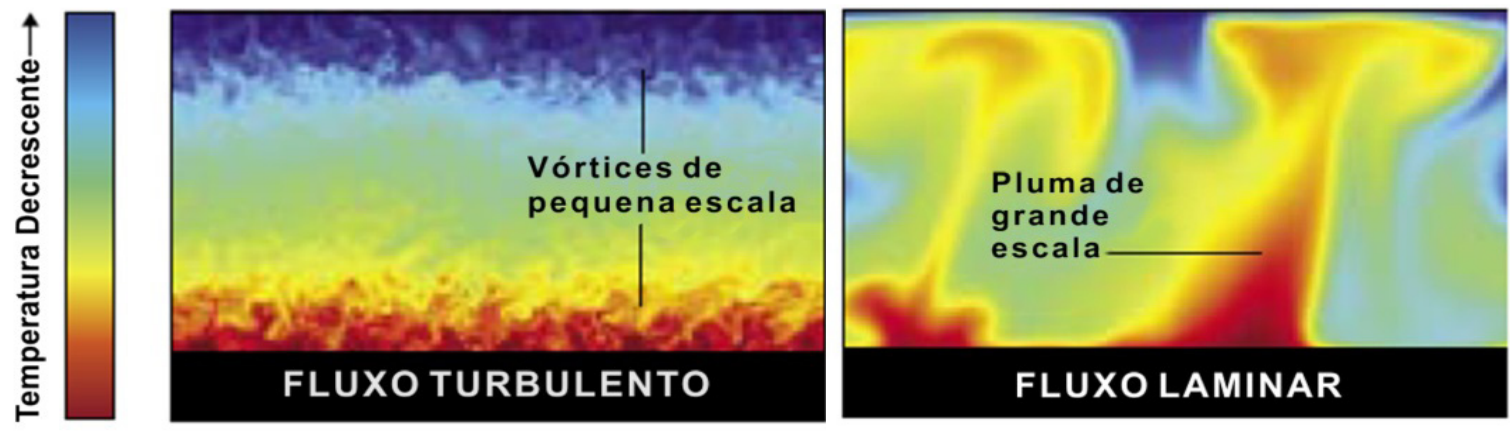

Figura 3. Padrões de convecção do fluido nos modelos computacionais de geodínamo, simulando a dinâmica do núcleo externo da Terra. A) Fluxo turbulento em pequena escala (modelo 2D), com dínamo não autossustentável e (B) fluxo laminar em grande escala (modelo 3D), com dínamo autossustentável. Fonte: Glatzmaier \& Olson (2005).

requer o uso de modelos bidimensionais (2D) e estes não conseguem manter um dínamo autossustentável (Glatzmaier \& Olson 2005). Com o desenvolvimento de computadores com melhores resoluções espaciais e maior velocidade no processamento de dados, talvez os pesquisadores consigam reduzir os valores das difusividades nos modelos em 3D de forma a simular a turbulência esperada (Glatzmaier 2007).

Nos modelos de geodínamo em laboratório, a maior dificuldade é a diferença entre o tamanho real do núcleo da Terra e o tamanho dos instrumentos utilizados. Por causa do pequeno tamanho destes últimos, é necessário que o fluido atinja velocidades muito altas para manter o dínamo autossustentável. Já no caso da Terra, devido a grande dimensão de seu núcleo, a velocidade do ferro fundido para manter o geodínamo é bem menor (Glatzmaier \& Olson 2005).

\section{Análise numérica dos principais parâmetros}

Os valores dos parâmetros adimensionais para as análises estatísticas aqui realizadas foram retirados dos artigos reunidos por Kono \& Roberts (2002). Estes autores pesquisaram 34 publicações e as agruparam com base nos valores semelhantes dos parâmetros, esquemas computacionais e equações usadas (Tabela 1).

É importante ressaltar que embora estes artigos utilizem os mesmos parâmetros adimensionais, uma comparação imediata entre os seus valores não é coerente, pois a definição desses parâmetros varia de autor para autor (Kono \& Roberts 2002). Por exemplo, enquanto $R a=\alpha g_{0} Q_{0} / 2 k \kappa \Omega$ para o grupo GR95, para o grupo KB97, $R a=\alpha \beta_{1} g_{0} R_{0}^{2} / 2 \eta \Omega$. Por isso, Kono \& Roberts (2002) adotam uma definição

Tabela 1. Publicações sobre modelos de geodínamo organizadas em grupos por Kono \& Roberts (2002).

\begin{tabular}{l|l}
\hline Grupo & Publicações \\
\hline KS95 & Kageyama et al. (1995), Kageyama e Sato (1997a,1997b,1997c), Ochi et al. (1999). \\
\hline GR95 & Glatzmaier \& Roberts (1995a,1995b). \\
\hline GR96 & $\begin{array}{l}\text { Glatzmaier \& Roberts (1996a, 1996b, 1997), Coe et al. (2000), Roberts e Glatzmaier (2000a), } \\
\text { Glatzmaier et al. (1999). }\end{array}$ \\
\hline KB97 & Kuang e Bloxham (1997,1998,1999), Bloxham (2000a, 2000b) \\
\hline KAK97 & $\begin{array}{l}\text { Kida et al. (1997), Kida \& Kitauchi (1998a,1998b), Kitauchi \& Kida (1998), } \\
\text { Ishihara \& Kida (2000) }\end{array}$ \\
\hline COG98 & Christensen et al. (1998, 1999, 2001), Olson et al. (1999), Kutzner \& Christensen (2000) \\
\hline SK99 & Sakuraba e Kono (1999), Kono et al. (2000b) \\
\hline GBT99 & Grote et al. (1999, 2000a, 2000b) \\
\hline KMH99 & Katayama et al. (1999) \\
\hline
\end{tabular}


Tabela 2. Parâmetros adimensionais usados nos modelos de geodínamo. Fonte: modificado de Kono \& Roberts (2002).

\begin{tabular}{l|l|l|l}
\multicolumn{1}{c|}{ Número } & Símbolo & \multicolumn{1}{c}{ Definição } & \multicolumn{1}{c}{ Razão } \\
\hline Rayleigh & $\mathrm{Ra}$ & $\alpha \beta_{0} \mathrm{~g}_{0} \mathrm{R}_{0}^{4} / v \kappa$ & Força de flutuabilidade e viscosidade \\
\hline Ekman & $\mathrm{E}$ & $v / 2 \Omega \mathrm{R}_{0}^{2}$ & Força de viscosidade e Coriolis \\
\hline Prandtl & $\operatorname{Pr}$ & $v / \kappa$ & Tempo de difusão viscosa e termal \\
\hline $\begin{array}{l}\text { Magnético de } \\
\text { Prandtl }\end{array}$ & $\mathrm{Pm}$ & $v / \eta$ & Tempo de difusão viscosa e magnética \\
\hline $\begin{array}{l}\text { Razão das difusi- } \\
\text { vidades }\end{array}$ & $\mathrm{q}$ & $\kappa / \eta$ & Tempo de difusão termal e magnética \\
\hline $\begin{array}{l}\text { Magnético de } \\
\text { Reynolds }\end{array}$ & $\mathrm{Rm}$ & $\mathrm{V}_{0} \mathrm{R}_{0} / \eta$ & Termo da advecção e difusão \\
\hline Elsasser & $\Lambda$ & $\mathrm{B}_{0}^{2} / 2 \Omega \eta \mu_{0} \rho_{0}$ & Força de Lorentz e Coriolis \\
\hline Rossby & $\mathrm{Ro}$ & $\mathrm{V}_{0} / \Omega \mathrm{R}_{0}$ & Força de inércia e Coriolis \\
\hline
\end{tabular}

Tabela 3. Valores dos parâmetros adimensionais padronizados e outras informações sobre os modelos de geodínamo de cada grupo. Fonte: modificado de Kono \& Roberts (2002).

\begin{tabular}{|c|c|c|c|c|c|c|c|c|c|c|}
\hline Parâmetros & KS95 & GR95 & GR96 & KB97 & KAK97 & COG98 & SK99 & GBT99 & КМH99 & Terra \\
\hline $\begin{array}{c}\text { Numero de } \\
\text { Rayleigh }\end{array}$ & $0,8-1,1 \cdot 10^{5}$ & $5,4.10^{12}$ & $4,6.10^{9}$ & $0,15-2,8 \cdot 10^{8}$ & $0,5-2,6.10^{5}$ & $0,07-1,5 \cdot 10^{7}$ & $1,0.10^{7}$ & $0,1-1,7 \cdot 10^{7}$ & $6,2-7,7.10^{3}$ & $10^{30}$ \\
\hline $\begin{array}{l}\text { Numero de } \\
\text { Ekman }\end{array}$ & $2,0.10^{-4}$ & $8,4 \cdot 10-7$ & $8,2 \cdot 10^{-7}$ & $2.0-10.10^{-5}$ & $1,9-2,8.10^{-3}$ & $6,3-63.10^{-5}$ & $3,2.10^{-5}$ & $3,6-21.10^{-5}$ & $3,6-36.10^{-3}$ & $10^{-15}$ \\
\hline $\begin{array}{c}\text { Numero de } \\
\text { Prandtl }\end{array}$ & $0,78-1$ & 5000 & 725 & 1 & 1 & 1 & 1 & 1 & 1 & 0,2 \\
\hline $\begin{array}{c}\text { Numero } \\
\text { magnético de } \\
\text { Prandtl }\end{array}$ & $10-28$ & 500 & 725 & 1 & $8,3-50$ & $1-5$ & 20 & $1-50$ & 35 & $5.10^{-6}$ \\
\hline $\begin{array}{c}\text { Razão das difu- } \\
\text { sividades }\end{array}$ & $12,82-28$ & 0,1 & 1 & 1 & $8,3-50$ & $1-5$ & 20 & $1-50$ & 35 & $2,5 \cdot 10^{-5}$ \\
\hline Razão dos raios & 0,30 & 0,351 & 0,351 & 0,343 & 0,5 & 0,351 & 0,4 & 0,4 & 0,4 & 0,351 \\
\hline $\begin{array}{c}\text { Mudança de } \\
\text { densidade }^{1}\end{array}$ & $\mathrm{C}$ & B & I & B & B & B & B & $\mathrm{B}$ & B & NA \\
\hline $\begin{array}{c}\text { Velocidade nas } \\
\text { condições de } \\
\text { contorno }\end{array}$ & No-slip & No-slip & No-slip & Stress-free & No-slip & No-slip & No-slip & Stress-free & No-slip & NA \\
\hline
\end{tabular}

padrão para cada parâmetro e em seguida padronizam as definições dos outros autores em relação as suas, obtendo então valores padronizados destes parâmetros ${ }^{10}$. As definições padrões usadas por Kono \& Roberts (2002) são iguais àquelas mostradas na Tabela 2 para $E, P r$ e $P m$, e para o $R a$ há uma pequena modificação, pois os autores acharam importante incluir a flutuabilidade composicional além da termal como fonte de convecção do núcleo externo. Após a padronização, Kono \& Roberts (2002) obtiveram a Tabela $3 \mathrm{com}$ as principais informações dos modelos dos grupos. É a partir das informações dessa tabela que as análises estatísticas deste artigo formam realizadas.

\footnotetext{
10 Para mais detalhes de quais são as definições dos parâmetros para cada um dos 9 grupos da Tabela 1 e como elas são padronizadas, consultar Kono e Roberts (2002).
}

Nas análises estatísticas, formam construídos três diagramas de dispersão e quatro histogramas (Figuras 4 a 7), sendo que para cada diagrama foram obtidos: i) gráfico da linha de tendência com sua equação da reta e valor de $\mathrm{R}^{2}$ (coeficiente de determinação), ii) gráfico da correlação dos dados iii) o valor da correlação linear entre os dados. A escolha da linha de tendência que melhor se ajustava aos dados foi feita com base nos valores mais elevados

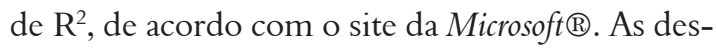
crições qualitativas das correlações lineares foram feitas segundo Callegari (2003 apud Lira 2004) sendo que: i) para valores entre 0,00 e 0,30 é fraca, ii) para valores entre 0,30 (incluindo-o) e 0,60 é moderada e iii) para valores entre 0,60 (incluindo-o) e 0,90 é forte. 


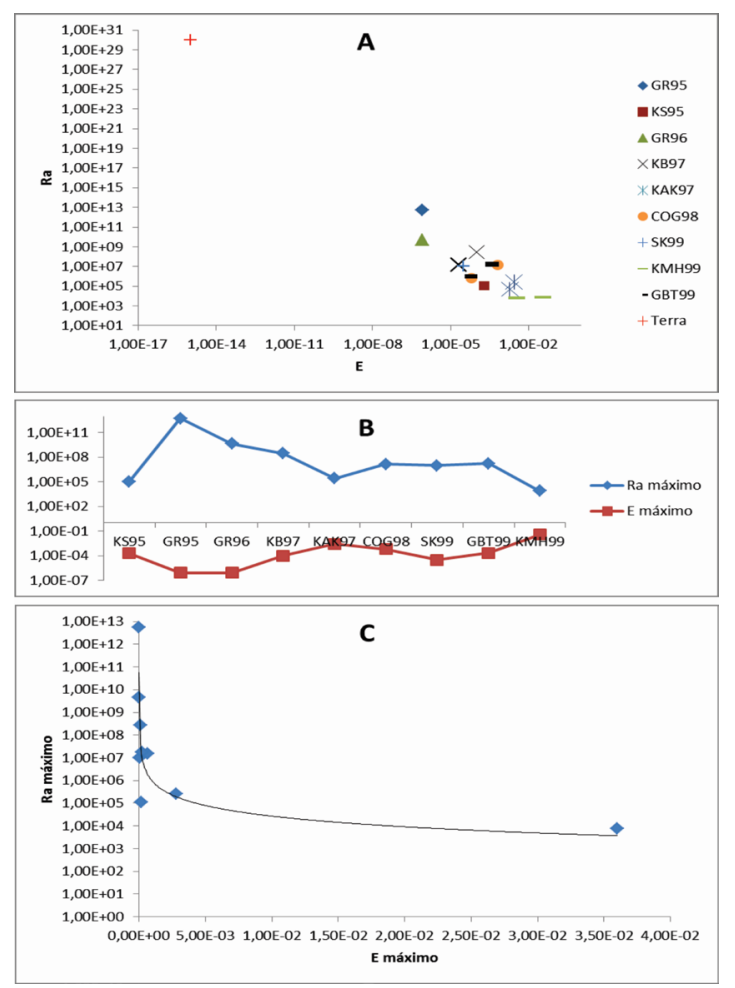

Figura 4. Diagrama de dispersão (A) modificado de Kono \& Roberts (2002), gráfico de correlação (B) e de linha de tendência $(C)$ de Ra $x E$.

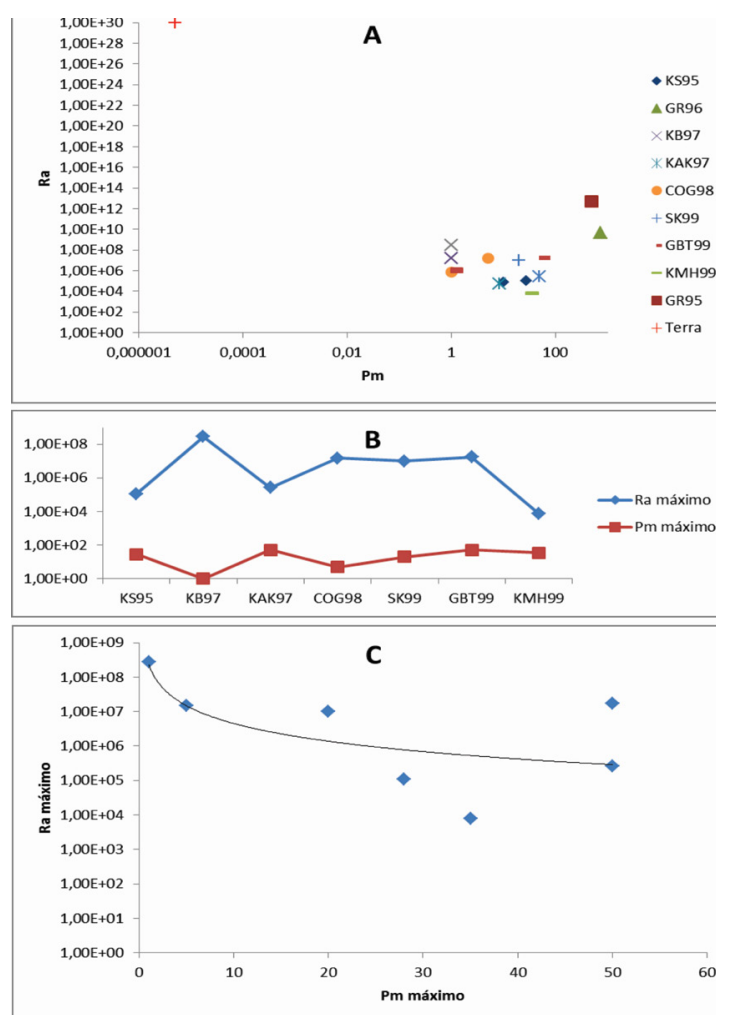

Figura 5. Diagrama de dispersão (A), gráfico de correlação (B) e de linha de tendência (C) de Ra x Pm.
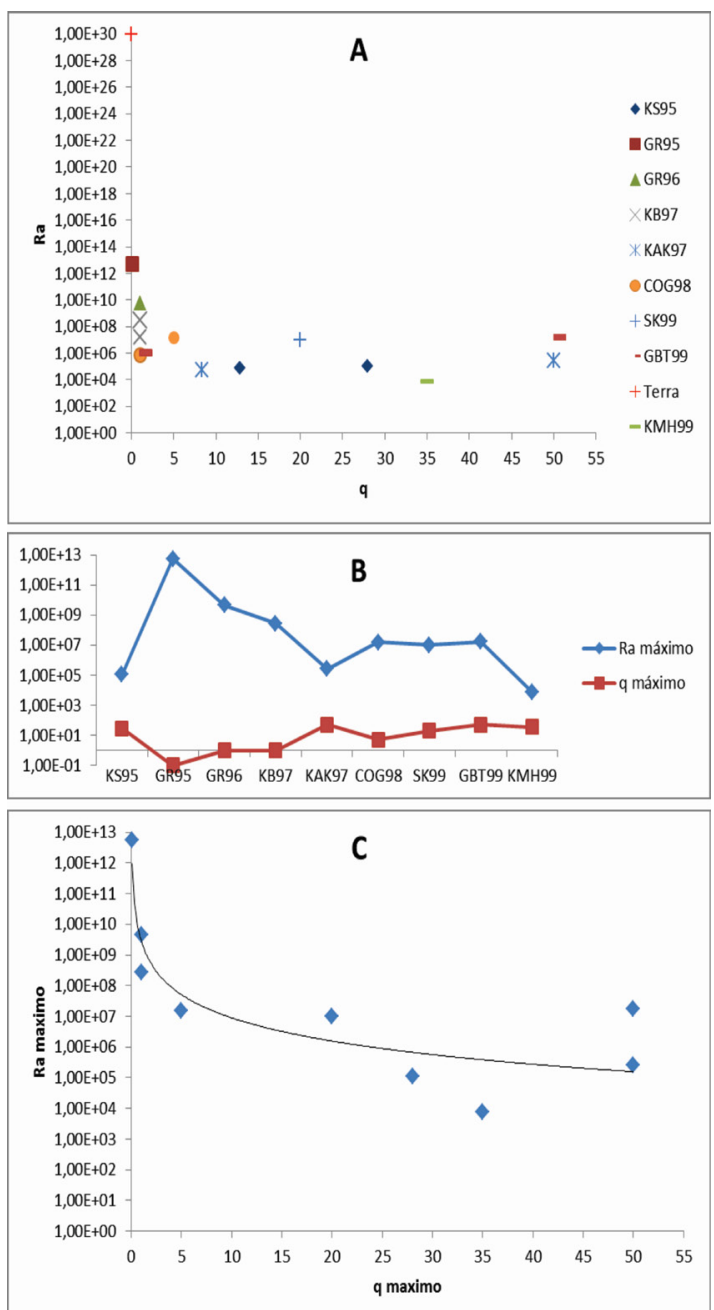

Figura 6. Diagrama de dispersão (A), gráfico de correlação (B) e de linha de tendência (C) de Ra x q.

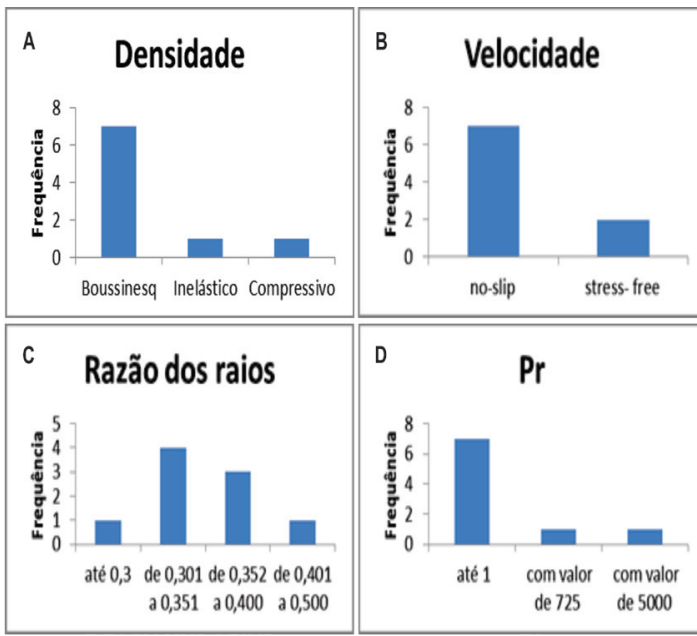

Figura 7. Histogramas das densidades do núcleo externo (A), velocidade nas condições de contorno (B), razão dos raios $R_{0} / R_{1}(C)$ e valores de $\operatorname{Pr}(D)$ usados nos modelos de geodínamo em MHD. 
Tabela 4. Resumo dos valores estatísticos dos diagramas de dispersão.

\begin{tabular}{l|l|l|l|l|l}
\hline \multicolumn{2}{c|}{} & $\begin{array}{l}\text { Correlação } \\
\text { linear }\end{array}$ & $\begin{array}{l}\text { Linha de } \\
\text { tendência }\end{array}$ & $\begin{array}{l}\text { Equação da } \\
\text { linha }\end{array}$ & $\mathbf{R}^{2}$ \\
\hline \multirow{2}{*}{$\operatorname{Ra} \times \mathrm{E}$} & $\begin{array}{l}\text { Valores } \\
\text { máximos }\end{array}$ & $-0,14$ & Potência & $\mathrm{y}=21,248 \mathrm{x}^{-1,551}$ & 0,7761 \\
\cline { 2 - 6 } & $\begin{array}{l}\text { Valores } \\
\text { mínimos }\end{array}$ & $-0,19$ & Potência & $\mathrm{y}=0,0157 \mathrm{x}^{-2,009}$ & 0,8354 \\
\hline \multirow{2}{*}{$\operatorname{Ra} \times \mathrm{Pm}$} & $\begin{array}{l}\text { Valores } \\
\text { máximos }\end{array}$ & $-0,60$ & Potência & $\mathrm{y}=2 \mathrm{E}+08 \mathrm{x}^{-1,708}$ & 0,4651 \\
\cline { 2 - 6 } & $\begin{array}{l}\text { Valores } \\
\text { mínimos }\end{array}$ & $-0,16$ & Potência & $\mathrm{y}=2 \mathrm{E}+06 \mathrm{x}^{-0,986}$ & 0,2824 \\
\hline \multirow{2}{*}{$\operatorname{Ra}$} & $\begin{array}{l}\text { Valores } \\
\text { máximos }\end{array}$ & $-0,38$ & Potência & $\mathrm{y}=3 \mathrm{E}+09 \mathrm{x}^{-2,512}$ & 0,819 \\
\cline { 2 - 6 } & $\begin{array}{l}\text { Valores } \\
\text { mínimos }\end{array}$ & $-0,28$ & Potência & $\mathrm{y}=1 \mathrm{E}+08 \mathrm{x}^{-2,665}$ & 0,6266 \\
\hline
\end{tabular}

bém, já que quanto menor for a viscosidade, maior deverá ser a força de flutuabilidade termal para superar $R a_{c}$. No caso da Terra, o fluido é o núcleo externo líquido e a presença da viscosidade nas camadas limitantes (CMB e ICB) é importante para diminuir o valor do $R a_{c}$ e permitir a convecção. No entanto, como a viscosidade é muito pequena no restante do núcleo externo, a presença do campo magnético nele

Todos os diagramas de dispersão contêm apenas os valores extremos dos parâmetros adimensionais (valores máximos e mínimos) de cada grupo. Embora os gráficos de linha de tendência e correlação correspondam apenas aos valores máximos, os dados estatísticos resumidos na Tabela 4 também incluem os valores mínimos. Os significados de todos os símbolos e notações utilizadas encontram-se na Tabela 5.

\subsection{Parâmetros adimensionais}

Assim como em mecânica de fluidos, as Equações (1), (2) e (3) utilizadas nos modelos em MHD são comumente reescritas em uma forma adimensional (Tabela 6), pois assim o fenômeno estudado fica caracterizado por um número pequeno de parâmetros adimensionais (Kono \& Roberts 2002). Um método possível de ser usado para tornar as variáveis adimensionais é adotando as unidades expressas na Tabela 7 .

Nas equações adimensionais da Tabela 6 aparecem 4 parâmetros adimensionais conhecidos como número de Rayleigh $(R a)$, número de $\operatorname{Ekman}(E)$, número de Prandtl $(P r)$ e número magnético de Prandtl $(\mathrm{Pm})$. Esses parâmetros, assim como a razão entre os raios do núcleo interno e externo $\left(R_{1} / R_{0}\right)$, são os principais dados de entrada nos modelos de geodínamo, caracterizando-os (Tabela 2).

O número de Rayleigh é a razão entre a força de flutuabilidade e a viscosidade. Se o seu valor é maior do que um determinado valor crítico $\left(R a_{c}\right)$ então o fluido transmitirá o calor pela convecção, e se for menor a transmissão será feita por condução apenas (Davidson 2001). A viscosidade em excesso inibe a convecção, no entanto a sua ausência tam- também tem papel importante para favorecer a convecção (Sreenivasan 2010). O valor estimado de $R a$ para a Terra é de $10^{30}$.

O número de Ekman é a razão entre a força de viscosidade e a força de Coriolis. No caso da Terra, o seu valor é $10^{-15}$ já que a força de Coriolis é muito mais expressiva no núcleo externo do que a viscosidade. O número de Prandtl é a razão entre os tempos de difusão viscosa e termal enquanto o número de magnético de Prandtl é a razão entre os tempos de difusão viscosa e magnética. A razão entre esses tempos de difusão é a razão das difusividades ou também conhecida como número de Roberts, obtido dividindo-se $\mathrm{Pm}$ por $\mathrm{Pr}$. Depois de realizadas as simulações, são obtidas quantidades que permitem definir três outros parâmetros adimensionais: os números de Elsasser $(\Lambda)$, Rossby (Ro) e o magnético de Reynolds ( $R m$ ) (Kono \& Roberts 2002). O número de Elsasser é a razão entre as duas principais forças atuantes no núcleo externo, a de Lorentz e a de Coriolis. Para valores de $\Lambda<1$, a força magnética tem intensidade menor que $10^{-3} \mathrm{~T}$, sendo tratada apenas como uma perturbação na dinâmica do fluido. Para estes modelos o dínamo é classificado como pertencente a um regime de campo fraco. Já para o caso da Terra, onde $\Lambda \geq 1$, a força magnética tem intensidade maior que $10^{-3} \mathrm{~T}$ sendo comparável com a de Coriolis. Para estes modelos o dínamo é classificado como pertencente a um regime de campo forte (Buffet 2002, Glatzmaier \& Roberts 1995b), tendo como exemplo os modelos de Glatzmaier \& Roberts (1995a, 1995b) e Kuang e Bloxham (1997). O número de Rossby é a razão entre as forças de inércia e de Coriolis, sendo em geral muito pequeno como é o caso de $R o=2,0.10^{-5}$ no modelo de Kuang e Bloxham (1997). 
Tabela 5. Significado dos símbolos usados ao longo do artigo e nas equações ${ }^{a}$. Fonte: modificado de Kono \& Roberts (2002)

\begin{tabular}{|c|c|c|}
\hline Símbolo & Significado & Unidade \\
\hline$\alpha$ & coeficiente de expansão termal & $\mathrm{K}^{-1}$ \\
\hline$g_{0}$ & gravidade & $\mathrm{m} \mathrm{s}^{-2}$ \\
\hline$Q_{0}$ & dissipação & $\mathrm{W} \mathrm{m} \mathrm{m}^{-3}$ \\
\hline$k$ & condutividade termal & $\mathrm{J} \mathrm{m}^{-1} \mathrm{~s}^{-1} \mathrm{~K}^{-1}$ \\
\hline$\kappa$ & difusividade termal & $\mathrm{m}^{2} \mathrm{~s}^{-1}$ \\
\hline$\Omega$ & taxa de rotação do manto & $\mathrm{s}^{-1}$ \\
\hline$\beta_{1}$ & gradiente de temperatura & $\mathrm{K} \mathrm{m}^{-1}$ \\
\hline$R_{0}$ & raio do núcleo externo & $\mathrm{m}$ \\
\hline$R_{1}$ & raio do núcleo interno & $\mathrm{m}$ \\
\hline$\eta$ & difusividade magnética & $\mathrm{m}^{2} \mathrm{~s}^{-1}$ \\
\hline$\rho$ & densidade & $\mathrm{kg} \mathrm{m}^{-3}$ \\
\hline$T$ & temperatura & $\mathrm{K}$ \\
\hline$t$ & tempo & $\mathrm{s}$ \\
\hline$P$ & pressão & $\mathrm{Pa}$ \\
\hline$v$ & $\begin{array}{l}\text { viscosidade cinemática ou } \\
\text { difusividade viscosa }\end{array}$ & $\mathrm{m}^{2} \mathrm{~s}^{-1}$ \\
\hline$\theta$ & pertubação na temperatura & $\mathrm{K}$ \\
\hline$\epsilon$ & $\begin{array}{c}\text { geração de calor por unida- } \\
\text { de de massa }\end{array}$ & $\mathrm{K} \mathrm{s}^{-1}$ \\
\hline $\boldsymbol{V}$ & velocidade & $\mathrm{m} \mathrm{s}^{-1}$ \\
\hline $\boldsymbol{B}$ & campo magnético & $\mathrm{T}$ \\
\hline$r$ & $\begin{array}{l}\text { vetor unitário radial ao } \\
\text { núcleo }\end{array}$ & \\
\hline $1 z$ & $\begin{array}{l}\text { vetor unitário paralelo ao } \\
\text { eixo de rotação do núcleo }\end{array}$ & \\
\hline
\end{tabular}

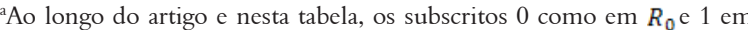
$\beta_{1}, R_{1}$ se referem aos valores dos símbolos no $C M B$ e ICB , respectivamente.

\subsection{Análises estatísticas}

O diagrama de dispersão de $R a \times E$ (Figura 4A) é importante porque estes dois parâmetros são os que melhor caracterizam os modelos de dínamos MHD em 3D (Kono \& Roberts 2002).A abscissa $E$ mede a velocidade de rotação sendo que quanto menor o $E$, maior é a força de Coriolis e, portanto maior a rotação do modelo. A ordenada $R a$ mede a quantidade de energia disponível ao modelo para iniciar a convecção: elevados valores de $R a$ significam que o modelo é movido por alta energia (Kono $\&$ Roberts 2002). Embora todos os grupos estejam longe do geodínamo real (Terra), o grupo GR95 é o que mais se aproxima enquanto o grupo que mais se distancia é o KMH99. A correlação linear entre estes dois parâmetros é fraca e negativa (Tabela 4) mostrando que quando o valor de $R a$ aumenta o de $E$ diminui e vice-versa. Essa oposição é confirmada visualmente pelo gráfico da correlação (Figura 4B). No entanto, a correlação mais adequada é não linear do tipo potência (Figura 4C) e sua linha de tendência indica que cerca de 77,61 a 83,54 \% da variância de $R a$ é explicado pela variância de $E$ (Tabela 4) .

O diagrama de $R a_{\times} P m$ (Figura 5A) mostra as táticas usadas pelos modeladores para atingir um dínamo autossustentável, na qual a indução magnética é superior ao decaimento do campo magnético. Uma delas consiste em usar valores altos de $P m$ em modelos que usam baixos valores de $R a$, próximo do $R a_{C}$ (Olson et al. 1999). Dessa forma, o alto valor de $\mathrm{Pm}$ reduz o tempo de difusão magnética $(\eta)$ em relação ao tempo de difusão viscosas $(v)$, aumentando o número magnético de Reynolds. De acordo com o diagrama, essa tática foi usada por KMH99 e KS95. A outra tática consiste em usar valores de $R a$ elevados ao invés de valores altos de $P m$ (Olson et al. 1999), assim como fez o grupo KB97. As exceções dessas táticas são GR95 e GR96, pois utilizam valores elevados tanto para $R a$ quanto para $\mathrm{Pm}$. O valor negativo das correlações lineares assim como o seu gráfico (Figura 5B) corroboram essas duas táticas, mostrando que, em geral, para valores altos de $R a$ os valores de $\mathrm{Pm}$ são baixos e vice-versa. A correlação linear é forte para os valores máximos e fraca para os valores mínimos, mas a linha de tendência que melhor se adequa aos dados é não linear do tipo potência (Figura $5 \mathrm{C}$ ), e o seu valor de $\mathrm{R}^{2}$ mostra que 28,24 a 46,51\% da variância de $R a$ é explicado pela variância de $\mathrm{Pm}$ (Tabela 4).

O diagrama de $R a \times q$ (Figura 6A) mostra outra estratégia usada pelos pesquisadores, mas o seu uso é feito para tentar atingir a parametrização do espaço apropriada para o geodínamo. Essa tática consiste em modelar a convecção com uma rotação lenta próxima ao $R a$ crítico, onde a corrente é periódica e de grande escala, e depois usar um valor alto para o número de Roberts $(q)$ para atingir as condições de um dínamo (Olson et al. 1999). Segundo o diagrama, os modelos 
que usam essa tática são KMH99, KS95 e KAK97 já que possuem os valores mais baixos de $R a$ e os mais elevados de $q$. A correlação linear é fraca a moderada e o seu valor negativo assim como o gráfico da correlação (Figura 6B) corroboram essa oposição entre os parâmetros $R a$ e $q$. A melhor correlação é não linear do tipo potência (Figura 6C), sendo que sua linha de tendência indica que 62,66 a 81,9 \% da variância de $R a$ é explicado pela variância de $q$ (Tabela 4).

O histograma da densidade (Figura 7A) mostra que a maioria dos modelos utiliza a aproximação de Boussinesq, mas há exceções como GR96 que utilizam a aproximação inelástica, considerando que a densidade na base do núcleo externo fluido é $20 \%$ maior do que no topo (Glatzmaier 2007). Quanto à razão dos raios $\mathrm{R}_{1} / \mathrm{R}_{0}$ (Figura $7 \mathrm{C}$ ), a maioria dos modelos usa o valor próximo ao da Terra $(0,351)$.

O histograma da condição de contorno para a velocidade (Figura 7B) mostra que a maioria dos modelos considera que as forças de viscosidade nas fronteiras $(I C B$ e $C M B)$ são relevantes, de forma que a velocidade do fluido nelas seja zero (no-slip) ao invés de considerá-las (stress-free). O uso de uma condição ou outra é muito relevante, pois modifica a circulação do fluido condutor no núcleo externo e gera um campo magnético diferente como foi verificado por Kuang e Bloxham (1997). Ao adotarem a condição stress-free no seu modelo, o fluido e a componente toroidal do campo magnético ficaram homogeneamente distribuídos por todo o núcleo externo. Mantendo todos os outros parâmetros fixos e modificando apenas a condição para no-slip, o fluido condutor se concentrou próximo ao eixo de rotação do sistema e o campo toroidal ficou concentrado no $I C B$.

De forma geral, os modelos adotam baixos valores de difusividades (termal, magnética e viscosa), o que resulta em um baixo valor de $\mathrm{Pr}$ como é visualizado no seu histograma (Figura 7D). No entanto, alguns grupos como GR95 e GR96 usam valores muito acima do esperado para a difusividade viscosa ${ }^{11}$ em função da escolha dos valores de $E$. Dessa forma, o valor de $\mathrm{Pr}$ para estes grupos é muito maior que o dos outros.

\section{Conclusões}

Os primeiros modelos computacionais de geodínamo eram cinemáticos, e buscavam apenas a compreensão de quais fluidos poderiam gerar um campo

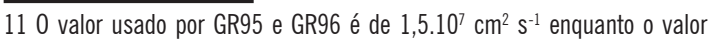
esperado no núcleo é de $10^{-2} \mathrm{~cm}^{2} \mathrm{~s}^{-1}$. magnético, sendo os fluidos arbitrariamente descritos. A lei de Gauss para campos magnéticos (Equação 3) e a equação de indução magnética (Equação 6) são as principais equações utilizadas nestes modelos, sendo que nesta última o termo de advecção prevalece sobre o de difusão. Dessa forma, o campo magnético do planeta não é exaurido pela dissipação ôhmica e as linhas do campo magnético são esticadas e arrastadas pelo ferro fundido no núcleo externo, de acordo com o Teorema de Alfvén, reforçando o campo original. O teorema de Cowling é refutado pelo funcionamento de um dínamo do tipo $\alpha \omega$, onde um campo poloidal gera um campo toroidal (efeito $\omega$ ) e este por sua vez gera um campo poloidal (efeito $\alpha$ ) fechando o ciclo.

Os modelos seguintes incorporam os efeitos do campo magnético no fluido e para isso usam a magnetohidrodinâmica. As principais equações utilizadas, além das Equações (3) e (6) são: a de Navier-Stokes (Equação 8) que descreve a influência das forças atuantes no fluido, a de continuidade (Equação 9) representando a incompressibilidade do fluido, e a de flutuabilidade (Equação 10) que move o fluido pela convecção termal e composicional. Nestes modelos, o núcleo externo é simulado como uma casca esférica entre o manto e o núcleo interno, contendo correntes meridionais simétricas e correntes zonais assimétricas em relação ao eixo de rotação da Terra. Embora haja critérios diferentes em cada modelo como, por exemplo, variações na densidade, fontes de flutuabilidade e condições de contorno, todos apresentam como resultado um campo dipolar fora do núcleo e correntes zonais para o oeste no CMB.

A principal dificuldade destes modelos computacionais é a impossibilidade de utilizar valores baixos de viscosidade para o fluido em 3D, o que acaba gerando um fluxo laminar de grande escala, diferente do que se espera no núcleo externo da Terra. Em compensação, os modelos em $2 \mathrm{D}$ permitem reduzir esses valores e gerar os vórtices de pequena escala esperados, no entanto não conseguem tornar o dínamo autossustentável. Em laboratório, a dificuldade está em colocar o fluido a uma velocidade alta o suficiente para compensar o pequeno tamanho dos aparelhos instrumentais, o que também torna o dínamo autossustentável. No futuro, com o aperfeiçoamento das resoluções espaciais e rapidez no processamento de dados dos computadores, os modelos em 3D talvez consigam utilizar valores reais de viscosidade e com isso gerar um fluxo turbulento de pequena escala.

Os principais diagramas de dispersão que representam os modelos de geodínamo são o de $R a_{\mathrm{X}} E$, 
$R a_{\mathrm{X}} q$ e $R a_{\mathrm{X}} P m$, sendo que os dois últimos mostram as táticas usadas para atingir um dínamo autossustentável e a parametrização do espaço apropriada para o geodínamo, respectivamente. Para todos eles, a melhor linha de tendência que se ajusta aos dados observados é do tipo potência com elevado valor do coeficiente de determinação (exceção para $R a_{\mathrm{X}}$ $\mathrm{Pm}$ ) e correlação linear é, em geral, fraca e negativa mostrando que quando um dos parâmetros aumenta o outro tende a diminuir. Os histogramas indicam que a maioria dos modelos utiliza: i) a densidade do núcleo externo constante com a aproximação de Boussinesq, ii) a velocidade nas condições de contorno como no-slip, iii) a razão dos raios parecida com o valor real da Terra e iv) valores de $P r$ até 1 .

\section{Agradecimentos}

Os autores agradecem ao Conselho Nacional de Desenvolvimento Científico e Tecnológico (CNPq) pela bolsa de iniciação científica por meio do Programa Institucional de Bolsas de Iniciação 2011/2012 (PIBIC).

\section{Referências Bibliográficas}

Braginsky S. I. 1963. Soviet Physics Doklady, 149:8 apud Glaztmaier, G. A., Roberts, P.H. 1998. Dynamo theory then and now. International Journal of Engineering Science, 36:1325-1338.

Buffet B. A. 2002. Earth's core and the geodynamo. Science, 288:2007-2012.

Callegari S. M. J. 2003. Bioestatística: princípios e aplicações. Porto Alegre: Artmed, 255p. apud Lira A. S. 2004. Análise de correlação: abordagem teórica e de construção dos coeficientes com aplicações. Curitiba: Univ. Fed. Paraná. 209p. (Dissert. Mestr. Ciências).

Davidson P.A. 2001. An Introduction to Magnetohydrodynamics. Cambridge: Cambridge Univ. Press. 452p.

Glatzmaier G. A., Roberts, P.H. 1995a. A three-dimensional convective dynamo solution with rotating and finitely conducting inner core and mantle. Physics of Earth and Planetary Interiors, 91:63-75.
Glatzmaier G.A., Roberts P.H. 1995b. A three-dimensional self-consistent computer simulation for a geomagnetic field reversal. Nature, 377:203-209.

Glatzmaier G.A. Roberts P.H. 1997. Simulating the geodynamo. Contemporary Physics, 38:269-288.

Glaztmaier G.A., Roberts P.H. 1998. Dynamo theory then and now. International Journal of Engineering Science, 36:1325-1338.

Glatzmaier G.A., Olson P. 2005. Sondando a complexidade do geodínamo. Scientific American, edição especial, 20:30-37.

Glatzmaier G.A. 2007. Geodynamo: numerical simulation. In: Encyclopedia of geomagnetism and paleomagnetism. Gubbis D., Herrero-Bervera E., eds. XXVI, 1054p. URL: http://www.springer.com/978-14020-3992-8. Acesso 10.01.2011.

Halliday D., Resnick R., Walker J. 2007. Fundamentos de Física. v. 3, $7^{\mathrm{a}}$ ed. Trad. Ronaldo Sérgio de Biasi. Rio de Janeiro: LTC Livros Técnicos e Cient. 380p.

Hollerbach R. 1996. On the theory of the geodynamo. Physics of the Earth and Planetary Interiors, 98:163-185.

Jacobs J.A. 1994. Reversals of the Earth's magnetic field. $2^{\text {a }}$ ed. Cambridge: Cambridge University Press. 346 p.

Kono M., Roberts P.H. 2002. Recent geodynamo simulations and observations of the geomagnetic field. Review of Geophysics, 40(4):1-53.

Kuang W., Bloxham J. 1997. An Earth-like numerical dynamo model. Nature, 389:371-374.

Lowrie W. 2007. Fundamentals of Geophysics. $2^{\mathrm{a}}$ ed. Cambrigde: Cambrigde Univ. Press. 381p.

Olson P., Christensen U., Glatzmaier G. A. 1999. Numerical modeling of the geodynamo: Mechanisms of field generation and equilibration. J. Geophys. Res., 104:10.383 -10.404.

Silva B.R.F.da. 2009. O Dínamo Terrestre e a Topografia da Interface Manto Núcleo. São Paulo: Inst. Astron., Geof. C. Atmosf. USP. 101p. (Dissert. Mestr. Geofísica).

Sreenivasan B. 2010. Modeling the geodynamo: progress and challenges. Current Sciences, 12(99):17391750.

Verhoogen J. 1961. Geophysical Journal of the Royal Astronomical Society, 4: 276, apud Glaztmaier G. A. Roberts P.H. 1998. Dynamo theory then and now. International Journal of Engineering Science, 36: 1325-1338.

Resumo: Um dos principais desafios da geofísica global é entender como a dinâmica do núcleo externo líquido da Terra funciona na geração e manutenção do campo geomagnético, por meio de teorias de geodínamo. 0 campo geomagnético é de extrema importância para a manutenção da vida no nosso planeta, visto que nos protege das partículas de alta energia provenientes do Sol, além de ser útil para fins de navegação. Por ser inacessível ao homem, esses estudos são baseados nos resultados obtidos pela modelagem do núcleo em laboratórios e computadores, simulando as condições e parâmetros do interior do planeta. Cada modelo tem suas singularidades, semelhanças e restrições. Nesse contexto, este artigo reúne as informações básicas sobre o funcionamento dos modelos de geodínamo publicados. Foi realizada uma revisão dos artigos internacionais e livros sobre 0 assunto, de forma a compilar, organizar e sintetizar essas informações. São explicados conceitos, teorias e principais equações que descrevem esses modelos e são apresentadas análises estatísticas realizadas com os principais parâmetros utilizados pelos modelos.

Palavras-Chave: campo geomagnético, modelos de geodinamo, magnetohidrodinâmica 